\title{
ChemComm
}

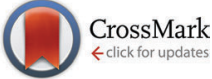

Cite this: Chem. Commun., 2015, 51, 5351

Received 27th October 2014

Accepted 11th January 2015

DOI: $10.1039 / c 4 c c 08472 a$

www.rsc.org/chemcomm

\section{A modular DNA origami-based enzyme cascade nanoreactor $\dagger$}

\author{
Veikko Linko, $\ddagger^{\mathrm{ab}}$ Marika Eerikäinen $\ddagger^{\mathrm{ab}}$ and Mauri A. Kostiainen ${ }^{\star^{a}}$
}

In this communication, we present a nanoscale reactor assembled from tuneable and spatially addressable tubular DNA origami units. We can controllably combine separate origami units equipped with glucose oxidase (GOx) and horseradish peroxidase (HRP), and demonstrate efficient GOx/HRP enzyme cascade reaction inside the tube. The reactor could be utilized as a nanoscale diagnostic tool, and modularity of the proposed system would further enable more complex reactions.

Nanoscale engineering has shown substantial potential to revolutionize a wide range of scientific fields making e.g. novel medical treatments ${ }^{1}$ and miniaturizing electronics possible. ${ }^{2}$ For biochemical applications, precise organization of materials on nanoscale could enable customized machinery that are able to mimic complex natural systems found in living cells. ${ }^{3}$ Numerous parallel multistep reactions can go on in the cells with exceptional efficiency and specificity including catalytic cycles. This is achieved via compartmentalization: ${ }^{3}$ enzymes are appropriately arranged in the micro-/nanoreactors, which control the flow of molecules through these domains and also separate different reaction compounds from each other.

Various materials and approaches can be used for encapsulating catalysts, such as sol-gel materials, ${ }^{4}$ and efficient catalytic reactions have been realized by utilizing for example porous polymersomes, ${ }^{5,6}$ carbon nanotubes, ${ }^{7,8}$ viruses, ${ }^{9,10}$ inorganic nanocrystal-protein complexes $^{11}$ and nanosized ferrous matrices ${ }^{12}$ as scaffolds for the catalysts. However, during recent years, the possibility to create exact and complex biocompatible nanoarchitectures by using DNA as a building material has markedly emerged. ${ }^{13-15}$ Especially the 'DNA origami' technique has become a widely

\footnotetext{
${ }^{a}$ Biohybrid Materials, Department of Biotechnology and Chemical Technology, Aalto University, FI-00076 Aalto, Finland.E-mail: mauri.kostiainen@aalto.fi

${ }^{b}$ Molecular Materials, Department of Applied Physics, Aalto University, FI-00076 Aalto, Finland

$\dagger$ Electronic supplementary information (ESI) available: Experimental details, additional TEM image of dimer formation, progress curves for DNA origami units, DNA origami designs and DNA strand sequences. See DOI: 10.1039/ c4cc08472a

\$ Equal contribution.
}

used method to fabricate arbitrary spatially well-controlled two- (2D) $)^{16}$ and three-dimensional (3D) nanostructures. ${ }^{17}$ The customized shapes and the nanoscale addressability of materials on DNA structures through rational design have yielded various interesting bionanotechnological applications including sophisticated drug delivery vehicles,${ }^{18}$ artificial ion channels ${ }^{19}$ gatekeepers for solid-state nanopores ${ }^{20-22}$ molecular scale electronic circuit boards ${ }^{23-25}$ and plasmonic devices. ${ }^{26,27}$

A DNA origami technique could be equally utilized in assembling enzyme systems for designed cascade reactions and studying the enzyme functions ${ }^{28}$ and reaction pathways. ${ }^{29}$ There exist a variety of examples of DNA-based enzyme systems ${ }^{30-32}$ but only the very recent approaches have taken advantage of the superior addressability of the DNA origami technique. These origami-based enzyme cascade arrangements include a distance-adjustable glucose oxidase (GOx)horseradish peroxidase (HRP) pair assembled on a rectangular origami ${ }^{33}$ and a similar system, where the $2 \mathrm{D}$ flat DNA sheet equipped with the enzyme pair was rolled into a confined tube, thus resulting in the encapsulation of the catalysts. ${ }^{34}$ Lately, a multi-enzyme reaction with a swinging arm geometry was built and demonstrated on a DNA-tile substrate. ${ }^{35}$

In this communication, we propose a modular enzyme cascade nanoreactor that is comprised of robust 3D DNA origami building blocks (see Fig. 1A). Each DNA origami unit can act as a building block hosting a chosen catalyst (or any other desired function). These blocks can be further controllably assembled together in any desired order thus forming a defined-size tubular nanofactory with a tailored assembly line. Here, we demonstrated the feasibility of the method by using two distinct units with either GOx- or HRP-enzymes anchored inside the origami compartment (Fig. 1B). The units were fabricated and purified separately, and efficiently glued together via programmable DNA base pairing. Finally, the catalytic activity of a two-unit nanoreactor was monitored in the environment containing D-glucose as a reactant and $3,3^{\prime}, 5,5^{\prime}$-tetramethylbenzidine (TMB) as a reporter (Fig. 1B).

The modular nanoreactor was assembled in a stepwise process, starting with the preparation of DNA origami building blocks. Two structurally different units were fabricated by annealing an M13mp18 


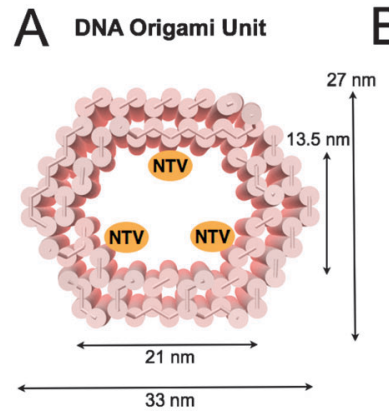

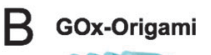
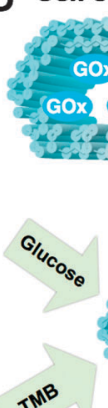

Fig. 1 (A) CanDo-simulated ${ }^{36}$ shape and dimensions of a DNA origami unit used as a building block for the nanoreactor. The length of an origami is approximately $30 \mathrm{~nm}$. NTVs indicate neutravidins, which are anchored to the inner surface of a tubular origami via biotinylated strands protruding from the origami. NTV acts as a binding site for biotinylated enzymes. (B) A schematic working principle of the nanoreactor. Two separately fabricated origami units are equipped with biotinylated glucose oxidase (GOx) or horseradish peroxidase (HRP) through biotin-avidin interaction. The units are linked together via base-pairing resulting in a nanoreactor that is able to perform an enzyme cascade reaction: (1) D-glucose enters the nanoreactor. In the presence of oxygen a hydrogen peroxide $\left(\mathrm{H}_{2} \mathrm{O}_{2}\right)$ is formed and released at the GOx enzyme site. (2) 3,3',5,5'-tetramethylbenzidine (TMB) is oxidized at the HRP enzyme as the diffused $\mathrm{H}_{2} \mathrm{O}_{2}$ is reduced to water. The formation of TMB diimine (TMB*) is detected using a spectrophotometer (absorbance at $650 \mathrm{~nm}$ ).

scaffold strand with the set of either 187 (GOx-origami) or 183 staple strands (HRP-origami). Each of the units contained 3 strands having biotin protruding from the inner surface of the tubular structure. NeutrAvidins (NTVs) were added to the units via biotin-avidin interaction in order to facilitate further binding of enzymes. A unit loaded with NTVs is presented in Fig. 1A. After NTVs were incorporated into the origamis, biotinylated enzymes (B-GOx or B-HRP) were attached to these units through the NTV binding sites (Fig. 1B). Between each step excess amounts of staple strands and unbound NTVs were removed by spin-filtering. In addition, an excess amount of HRP was removed using the same technique. See ESI $\dagger$ for the details.

The formed GOx- and HRP-origamis were connected together by hybridizing 32 short (3-6 bases) sequences. The short sequences sticking out at the end of one unit were paired with free scaffold sites located at the edge of another unit. ${ }^{37}$ In order to prevent the formation of multimers, the other end of the origami unit was passivated by overhanging single-stranded poly-T sequences (TTTTTTTT). Transmission electron microscopy (TEM) images of monomer units and the dimers assembled from the equal amounts of monomers within 1 day incubation at room temperature are shown in Fig. 2A and B. Fig. 2C displays agarose gel electrophoresed monomers and dimers revealing a high yield of the dimer formation (after the incubation of monomers, nearly $90 \%$ of all objects observed under TEM were correctly formed dimers, see ESI $\dagger$ ). By choosing the strands that connect the units uniquely, this programmable method could be generalized to well-defined modular multimers, thus enabling customized and more complex assembly lines.

Before studying the catalytic activity of a dimer nanoreactor, the units with enzymes were tested separately. In the experiments, the
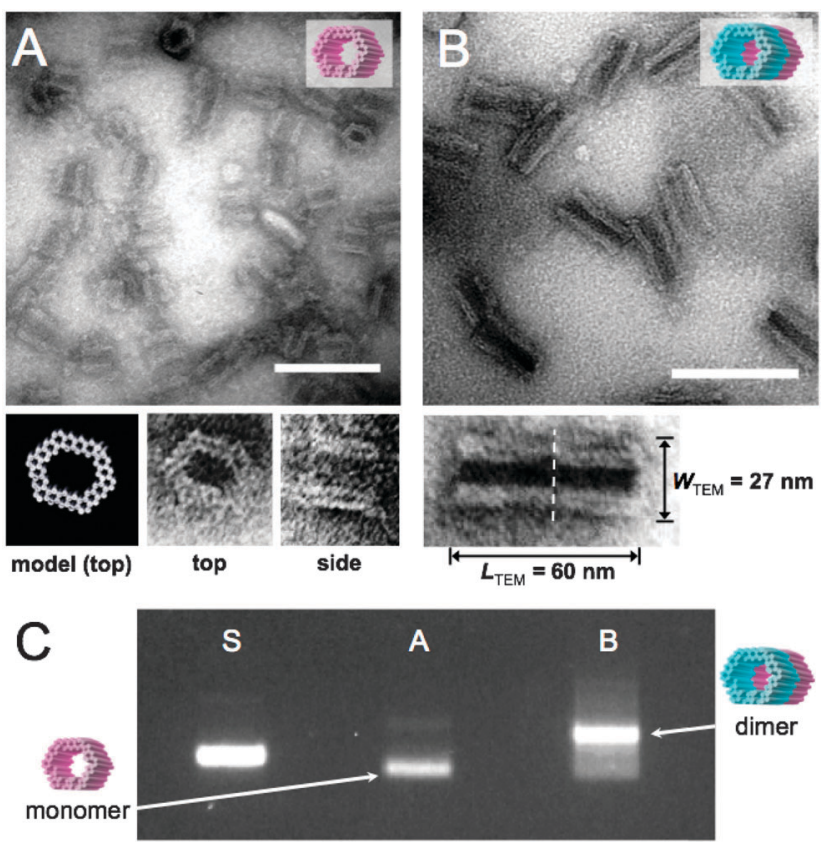

Fig. 2 (A) TEM micrograph of single DNA origami units. Close-up images of two orthogonal orientations (top and side) of the DNA origami unit correspond well with the CanDo ${ }^{36}$-predicted model of the structure. (B) TEM micrograph of dimer nanoreactors. Close-up image of a dimer shows the interface of two units (dashed line) and the dimensions of the dimer. After 1 day incubation of monomer units, $86 \%$ of all the observed objects were correctly formed dimers (calculated from TEM images, see ESI $\dagger$ ). Scale bars in $A$ and $B$ are $100 \mathrm{~nm}$. (C) Agarose gel electrophoresis: ' $S$ ' indicates M13mp18 scaffold reference, lane ' $A$ ' contains single origami units (monomers) and ' $B$ ' is two units attached to each other (dimers), similarly as in subfigures (A) and (B). Monomers are decently folded (lane A) and the intense additional band in lane $\mathrm{B}$ indicates a high yield of dimer formation.

concentration increase of the final product TMB $^{*}$ (see Fig. 1B) produced by a purified (spin-filtered) DNA origami unit equipped with either HRP (substrate containing TMB and $\mathrm{H}_{2} \mathrm{O}_{2}$ ) or GOx (substrate with sodium acetate, TMB, D-glucose and B-HRP) was characterized. The activity of these units was compared to samples that were fabricated and treated similarly but did not contain NTV binding sites. The results indicate that both units are indeed able to catalyze reactions, and furthermore, that the units show significantly higher maximum reaction rates than the controls (see ESI $\dagger$ for initial rates and Fig. 4 for details).

After the performance of single units was verified, the activity of the dimer nanoreactor equipped with the GOx-HRP cascade was explored by mixing the dimer solution (initial concentration $\sim 1 \mathrm{nM}$, final concentration in the measurement $\sim 100 \mathrm{pM}$ ) with sodium acetate-based ( $\mathrm{pH} 5,2.5 \mathrm{mM}$ ) substrate containing TMB $(250 \mu \mathrm{M})$ and D-glucose $(20 \mathrm{mM})$. The reactant glucose and the reporter TMB were added in excess amounts in order to achieve a reaction that is restricted by the diffusion rate of the intermediate product $\mathrm{H}_{2} \mathrm{O}_{2}$. The activity of the purified dimer nanoreactor (excess amount of HRP removed by spin-filtering) was compared to the reference dimer, which did not contain NTV binding sites (the samples were treated identically with enzymes and equally spin-filtered) (see Fig. 3). In addition, just the substrate without any enzymes or origamis (blank sample) was used as a control. 


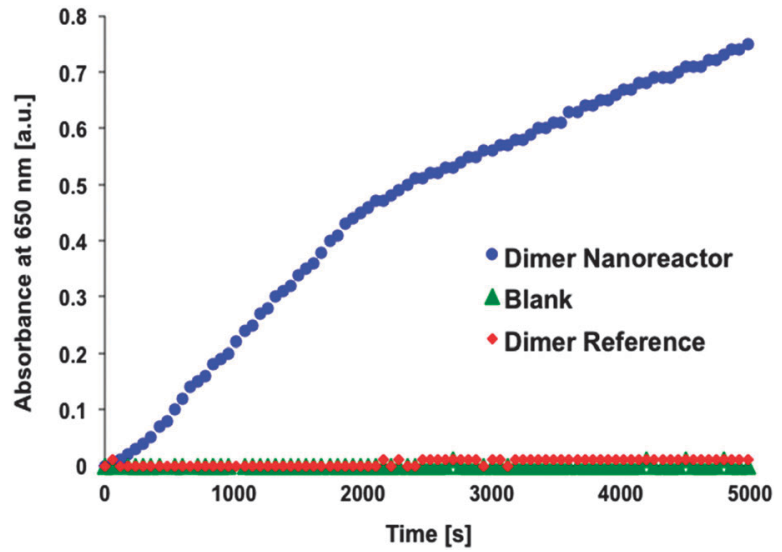

Fig. 3 Typical progress curves. Product concentration (absorbance of TMB* in arbitrary units) as a function of time for enzymatic dimer nanoreactor (blue dots), dimer reference reactor (dimer fabricated similarly but without NTVs, red diamonds) and an enzyme substrate only (blank sample, green triangles). The data shows that the activity of the assembled nanoreactor can clearly outdo the activity of the reference dimer. Reference does not indicate any significant activity but rather follows the progress curve of a blank sample. In this measurement $40 \mu \mathrm{l}$ of dimer samples (approx. $1 \mathrm{nM}$ ) were mixed with $260 \mu \mathrm{l}$ of the substrate $(3.5 \mathrm{mM}$ sodium acetate $(\mathrm{pH}$ 5), $150 \mu \mathrm{M}$ TMB and $10 \mathrm{mM}$ D-glucose) resulting in the final concentration of $\sim 100 \mathrm{pM}$ of origamis.

The results show that the assembled nanoreactor has significantly higher activity than the blank control sample, similar to that reported in previous studies. ${ }^{33,34}$

Our experiments additionally prove that unspecific binding between enzymes and origami structures is insignificant, since the dimer fabricated without NTV binding sites (dimer reference sample) shows negligible catalytic activity. The same trend can be clearly seen when the maximum rate of reactions for the single origami units is compared to the reference samples (see Fig. 4). Both origami units equipped with enzymes can outdo the activity of the reference samples, and the effect is even more pronounced in the case of a dimer nanoreactor. The purity of the nanoreactor is undoubtedly an important feature for the attainable diagnostic uses, and here we demonstrate that it can be achieved via a straightforward purification step of the origami units; unbound HRPs can be efficiently removed from the solution by spin-filtering (see also ESI $\dagger$ ). Therefore, by taking into account the high yield of dimer formation and the absence of free HRPs in the solution, the observed catalytic activity of the nanoreactor sample is predominantly resulting from the enzyme cascades located inside the properly assembled dimers. In addition, compared to previous studies, ${ }^{33,34}$ we have managed to significantly reduce the background activity of the free enzymes by purifying the units.

In summary, we have reported the successful formation of a modular DNA-origami-based nanoreactor that can efficiently perform a designed enzyme cascade reaction. We believe that the presented method could be equally used for more complex reactions since the number of the units in the reactor is not limited. Moreover, the compartmentalization of the enzymes inside the robust tubular origami could presumably enhance the molecular reaction rates similar to that previously observed for more flexible origami tubes. ${ }^{34}$ That would be the case especially

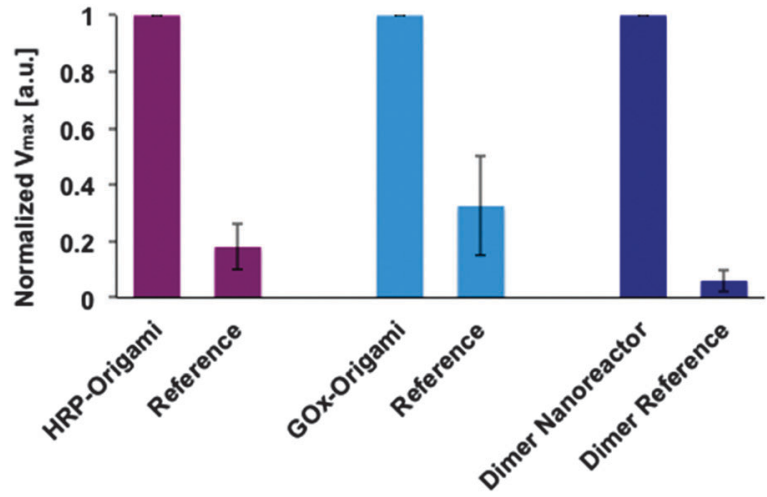

Fig. 4 Maximum rate of reactions $\left(V_{\max }\right)$ at the studied substrate concentration for enzymes attached to DNA origami units and for the assembled dimer nanoreactor. The maximal rate of reaction (formation of $\mathrm{TMB}^{*}$ ) for the sample is normalized to 1 in each case (for independent samples), and the performance of the sample is compared to a reference, which is fabricated and treated similarly but does not contain NTV binding sites. The DNA origamis were mixed in a 1:100-1:10 ratio with the substrate (final DNA origami concentration was typically $\sim 100 \mathrm{pM}$ in each measurement, see $\mathrm{ESI} \dagger$ for the details).

for larger molecules unable to diffuse through the barriers of the origami unit. The nanoreactor could be considered analogous to porous zeolites (molecular sieves) and on the other hand, the nanoreactor could efficiently process materials similar to holoenzymes. In addition, the tubular DNA vessels could be used for transporting cargo or an incorporated functional device into cells. This could be realized e.g. via virus ${ }^{38}$ or lipid bilayer ${ }^{39}$ encapsulation of DNA units. Thus, the proposed system could open up a cornucopia of opportunities for intriguing applications in synthetic biology and bionanotechnology.

We thank Christian Wachauf and Hendrik Dietz for discussions. Financial support from the Academy of Finland (grants 263504, 267497, 273645), Biocentrum Helsinki and Emil Aaltonen Foundation is gratefully acknowledged. This work was carried out under the Academy of Finland's Centres of Excellence Programme (2014-2019) and made use of the Aalto University Nanomicroscopy Centre (Aalto NMC).

\section{Notes and references}

1 M. M. Stevens and J. H. George, Science, 2005, 310, 1135-1138.

2 W. Lu and C. M. Lieber, Nat. Mater., 2007, 6, 841-850.

3 D. M. Vriezema, M. Comellas Aragonès, J. A. A. W. Elemans, J. J. L. M. Cornelissen, A. E. Rowan and R. J. M. Nolte, Chem. Rev., 2005, 105, 1445-1489.

4 D. Avnir, T. Coradin, O. Lev and J. Livage, J. Mater. Chem., 2006, 16, 1013-1030.

5 D. M. Vriezema, P. M. L. Garcia, N. S. Oltra, N. S. Hatzakis, S. M. Kuiper, R. J. M. Nolte, A. E. Rowan and J. C. M. van Hest, Angew. Chem., Int. Ed., 2007, 46, 7378-7382.

6 S. F. M. van Dongen, M. Nallani, J. J. L. M. Corneliessen, R. J. M. Nolte and J. C. M. van Hest, Chem. - Eur. J., 2009, 15, 1107-1114.

7 X. Pan, Z. Fan, W. Chen, Y. Ding, H. Luo and X. Bao, Nat. Mater., 2007, 6, 507-511.

8 T. W. Chamberlain, J. H. Earley, D. P. Anderson, A. N. Khlobystov and R. A. Bourne, Chem. Commun., 2014, 50, 5200-5202.

9 N. Carette, H. Engelkamp, E. Akpa, S. J. Pierre, N. R. Cameron, P. C. M. Christianen, J. C. Maan, J. C. Thies, W. Weberskirch, 
A. E. Rowan, R. J. M. Nolte, T. Michon and J. C. M. van Hest, Nat. Nanotechnol., 2007, 2, 226-229.

10 V. Liljeström, J. Mikkilä and M. A. Kostiainen, Nat. Commun., 2014, $5,4445$.

11 Z. Li, Y. Zhang, Y. Su, P. Ouyang, J. Ge and Z. Liu, Chem. Commun., 2014, 50, 12465-12468.

12 Q. Fu, W.-X. Li, Y. Yao, H. Liu, H.-Y. Su, D. Ma, X.-K. Gu, L. Chen, Z. Wang, H. Zhang, B. Wang and X. Bao, Science, 2010, 328, 1141-1144.

13 N. C. Seeman, Annu. Rev. Biochem., 2010, 79, 65-87.

14 V. Linko and H. Dietz, Curr. Opin. Biotechnol., 2013, 24, 555-561.

15 F. Zhang, J. Nangreave and H. Yan, J. Am. Chem. Soc., 2014, 136, 11198-11211.

16 P. W. K. Rothemund, Nature, 2006, 440, 297-302.

17 S. M. Douglas, H. Dietz, T. Liedl, B. Högberg, F. Graf and W. M. Shih, Nature, 2009, 459, 414-418.

18 S. M. Douglas, I. Bachelet and G. M. Church, Science, 2012, 335, 831-834.

19 M. Langecker, V. Arnaut, T. G. Martin, J. List, S. Renner, M. Mayer, H. Dietz and F. C. Simmel, Science, 2012, 338, 932-936.

20 N. A. W. Bell, C. R. Engst, M. Ablay, G. Divitini, C. Ducati, T. Liedl and U. F. Keyser, Nano Lett., 2012, 12, 512-517.

21 R. Wei, T. G. Martin, U. Rant and H. Dietz, Angew. Chem., Int. Ed., 2012, 124, 4948-4951.

22 C. Plesa, A. N. Ananth, V. Linko, C. Gülcher, A. J. Katan, H. Dietz and C. Dekker, ACS Nano, 2014, 8, 35-43.

23 V. Linko, S.-T. Paasonen, A. Kuzyk, P. Törmä and J. J. Toppari, Small, 2009, 5, 2382-2386.

24 H. T. Maune, S.-P. Han, R. D. Barish, M. Bockrath, W. A. Goddard III, P. W. K. Rothemund and E. Winfree, Nat. Nanotechnol., 2010, 5, 61-66.

25 V. Linko, J. Leppiniemi, S.-T. Paasonen, V. P. Hytönen and J. J. Toppari, Nanotechnology, 2011, 22, 275610.
26 A. Kuzyk, R. Schreiber, Z. Fan, G. Pardatscher, E.-M. Roller, A. Högele, F. C. Simmel, A. O. Govorov and T. Liedl, Nature, 2012, 483, 311-314.

27 A.-P. Eskelinen, R. J. Moerland, M. A. Kostiainen and P. Törmä, Small, 2014, 10, 1057-1062.

28 R. Subramani, S. Juul, A. Rotaru, F. F. Andersen, K. V. Gothelf, W. Mamdouh, F. Besenbacher, M. Dong and B. R. Knudsen, ACS Nano, 2010, 4, 5969-5977.

29 F. C. Simmel, Curr. Opin. Biotechnol., 2012, 23, 516-521.

30 O. I. Wilner, Y. Weizmann, R. Gill, O. Lioubashevski, R. Freeman and I. Willner, Nat. Nanotechnol., 2009, 4, 249-254.

31 M. Erkelenz, C.-H. Kuo and C. M. Niemeyer, J. Am. Chem. Soc., 2011, 133, 16111-16118.

32 S. Juul, F. Iacovelli, M. Falconi, S. L. Kragh, B. Christensen, R. Frøhlich, O. Franch, E. L. Kristoffersen, M. Stougaard, K. W. Leong, Y.-P. Ho, E. S. Sørensen, V. Birkedal, A. Desideri and B. R. Knudsen, ACS Nano, 2013, 7, 9724-9734.

33 J. Fu, M. Liu, Y. Liu, N. W. Woodbury and H. Yan, J. Am. Chem. Soc., 2012, 134, 5516-5519.

34 Y. Fu, D. Zeng, J. Chao, Y. Jin, Z. Zhang, H. Liu, D. Li, H. Ma, Q. Huang, K. V. Gothelf and C. Fan, J. Am. Chem. Soc., 2013, 135, 696-702.

35 J. Fu, Y. R. Yang, A. Johnson-Buck, M. Liu, Y. Liu, N. G. Walter, N. W. Woodbury and H. Yan, Nat. Nanotechnol., 2014, 9, 531-536.

36 C. E. Castro, F. Kilchherr, D.-N. Kim, E. L. Shiao, T. Wauer, P. Wortmann, M. Bathe and H. Dietz, Nat. Methods, 2011, 8, 221-229.

37 R. Jungmann, M. Scheible, A. Kuzyk, G. Pardatscher, C. E. Castro and F. C. Simmel, Nanotechnology, 2011, 22, 275301.

38 J. Mikkilä, A.-P. Eskelinen, E. H. Niemelä, V. Linko, M. J. Frilander, P. Törmä and M. A. Kostiainen, Nano Lett., 2014, 14, 2196-2200.

39 S. D. Perrault and W. M. Shih, ACS Nano, 2014, 8, 5132-5140. 